\title{
AN EMBEDDED SYSTEM FOR NON-INVASIVE BLOOD GLUCOSE MEASUREMENT IN GLUCOSE TOLERANCE TEST
}

\author{
Indhulekshmi M C $\mathbf{C}^{\mathbf{1}}$, Liju Philip ${ }^{2}$ \\ ${ }^{I} P G$ Scholar, Department of Electronics Engineering, College of Engineering Chengannur, Kerala, India \\ ${ }^{2}$ Assistant Professor, Department of Electronics Engineering, College of Engineering Chengannur, Kerala, India
}

\begin{abstract}
Diabetic patients need to monitor their blood glucose levels frequently to prevent complications related to the disease. Commonly used glucose measurement methods are invasive, uncomfortable, and usually require frequent finger pricking. This paper deals with the development of a non-invasive system for continuous measurement of blood glucose concentration levels, using near infrared light of suitable wavelength. Glucose determination using near infrared can be done by taking measurement of transmitted light through human body. This device may become very useful while conducting glucose tolerance test (GTT). In glucose tolerance test, patient should be given a measured dose of glucose solution to drink and blood is drawn at intervals of half an hour or one hour for two to four hours. This requires five to nine finger pricking for measurement of glucose concentration. By using this proposed device pricking can be reduced to one. First the glucose concentration is measured using conventional method for calibration. Then by comparing with the first sample measurement subsequent values can be determined without further pricking.
\end{abstract}

Keywords: Non invasive measurement, Near Infrared, Blood Glucose, Transmittance, Diabetes

\section{INTRODUCTION}

Diabetes is metabolic disorder in which blood glucose fluctuates from its normal range $(70-110 \mathrm{mg} / \mathrm{dl})$. Insulin is a hormone produced in body to regulate blood glucose level naturally. Under some pathological failure, body is not able to produce insulin or body cells become unable to use insulin. Thus amount of glucose present in blood exceeds normal range and patient may become diabetic. Nowadays number of diabetic people is increasing across the world due to population growth, unhealthy diet, obesity and lack of physical activity. Diabetic patients need to monitor their blood glucose levels frequently to prevent complications related to the disease.

Most of commercially available glucose measurement devices are invasive. The invasive methods are painful, have high recurring cost and danger of spreading infectious diseases. Non-invasive methods are more desirable and excellent alternatives to these devices. Non-invasive glucose monitoring could make millions of people more relaxed and comfortable during blood glucose testing. Thus it is necessary to develop a non-invasive blood glucose measurement system which is painless, convenient and cost effective. Non-invasive monitoring system will be a major breakthrough in the area of treating diabetes patients. Optical methods are painless and promising methods that can be used for non-invasive blood glucose measurement. Near-infrared (NIR) is one of the most widely explored optical techniques because of its high penetration in skin.

This technique has been applied on various body parts: finger, palm, arm, forearm, earlobe etc.
This work deals with the development of a device for measuring glucose concentration using near infrared light. The proposed device may become very useful while conducting glucose tolerance test (GTT).Glucose tolerance test (GTT) is a medical test which measures how well our body is able to break down glucose. In this test patient is given a measured dose of glucose solution to drink. Then blood samples are taken at specified intervals for the measurement of glucose concentration to determine how quickly it is cleared from the blood. Usually Glucose tolerance test takes about two to four hours. The patient is instructed to fast (water is allowed) for eight to twelve hours prior to the test. First a fasting blood sample is taken and then patient is given a measured dose of glucose solution to drink. Blood is drawn at intervals of half an hour or one hour for two to four hours to determine the glucose concentration. This requires five to nine finger pricking which cause pain and discomfort to patients.

By using this proposed device pricking can be reduced to one. First the glucose concentration is measured using conventional method for calibration. Then by comparing with the first sample measurement subsequent values can be determined without further pricking.

The goal of this work is to develop a reliable, simple, painless and portable device for glucose measurement that does not cause repetitive puncturing of skin.

\section{PROPOSED SYSTEM}

Near infrared based optical technique has been selected from a number of methods because it is easier to implement and needs less complex hardware setup.The near infrared 
(NIR) light in the range of $700 \mathrm{~nm}-2500 \mathrm{~nm}$ is the most suitable for detecting glucose concentration.

The proposed system is based on the principle of transmittance measurement. The value of transmission of light energy is dependent on the number of molecules present in absorbing material. Thus, intensity of light energy transmitted out from the absorbing substance is used as a sign of concentration of that particular substance. Hence by measuring intensity of transmitted light through the measurement site, concentration of glucose in blood can be calculated.

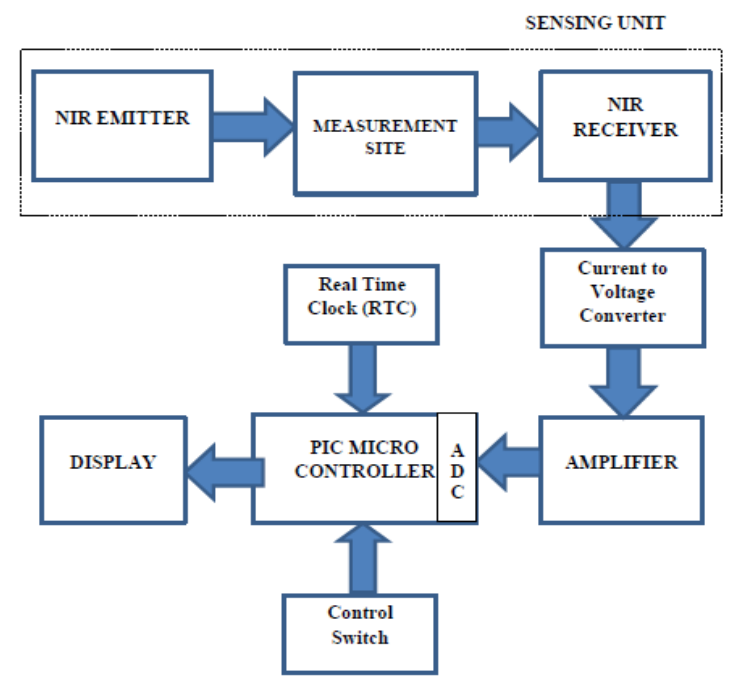

Fig-1: Non invasive glucose concentration measurement system

This system allows continuous measurement of variation in blood glucose concentration. The instrumentation for optical measurements (sensing unit) consists of an NIR emitter and receiver of $950 \mathrm{~nm}$ wavelength which is suitable for glucose concentration measurement.NIR rays emitted by the NIRLED passes through the measurement site and the transmitted rays are received by NIR receiver. After amplification output signal from the sensing unit is given to the microcontroller. The microcontroller has an inbuilt analog to digital converter that converts the analog electrical signal into digital values. The digitized values are then processed in microcontroller to determine the corresponding blood glucose concentration.

For proper measurement site selection two factors should be considered: i) The measurement site should be easily accessible ii) NIR light should easily transmit through the measurement site. Hence earlobe or finger web is selected as suitable measurement site.

The sensing unit must be fixed in proper position during entire measurement period. It is designed in such a way that the detector is placed exactly opposite and in line to the NIR light source for detecting maximum amount of transmitted light. The light source and detector should be placed as close as possible to the measurement site in physical contact with the skin.

\subsection{Operation of the System}

First glucose concentration is measured using conventional invasive method. After calibrating the proposed device with the first sample measurement, subsequent glucose concentration values are determined without further finger pricking. Thus pricking can be reduced to one while conducting glucose tolerance test.

Once the system is turned on, the infrared light propagates through the measurement site. During the propagating phase, NIR light interacts with the molecules present in the medium (glucose in blood). A part of light gets absorbed depending on the glucose concentration of blood under test and remaining part is transmitted through the measurement site. The transmitted signal is then detected by the infrared sensitive detector. After amplification the signal is digitized and is processed in the microcontroller to determine the blood glucose concentration. The measured blood glucose concentration is then displayed on the LCD. The time of measurements and the corresponding value of glucose concentration are stored in memory for further analysis

\section{RESULTS AND DISCUSSION}

The design was tested on seven non-diabetic individuals including both genders. The measurement sites chosen were earlobe and part of skin between thumb \& index finger. The near infrared emitter and receiver were positioned exactly opposite to each other to obtain transmitted signal through measurement site.

First the proposed device is calibrated with fasting glucose concentration obtained by the invasive method. After meal the signal from the detector is taken every half an hour for two to three hours to determine the variation in glucose concentration. The persons were not allowed to move throughout the measurement period to avoid change in sensor unit positioning in the measurement site.

Table-1: Fasting glucose value obtained from invasive

\begin{tabular}{|l|l|l|l|l|l|l|l|}
\multicolumn{8}{|c}{ laboratory test } \\
\hline $\begin{array}{l}\text { Person } \\
\text { glucose }(\mathrm{mg} / \mathrm{dl})\end{array}$ & 89 & 85 & 90 & 87 & 91 & 100 & 85 \\
\hline
\end{tabular}

Table-2: Variation in glucose concentration obtained from

\begin{tabular}{|c|c|c|c|c|c|}
\multicolumn{6}{c|}{ proposed non invasive device after meal } \\
\hline \multirow{2}{*}{ Person } & $\begin{array}{c}\text { Measurement } \\
\text { site }\end{array}$ & \multicolumn{2}{|c|}{ Variation in glucose concentration (mg/dl) with time } \\
\cline { 3 - 6 } & & $30 \mathrm{~min}$ & $60 \mathrm{~min}$ & $90 \mathrm{~min}$ & $120 \mathrm{~min}$ \\
\hline A & Finger web & 91 & 96 & 100 & 105 \\
\hline B & Finger web & 88 & 94 & 97 & 100 \\
\hline C & Finger web & 94 & 102 & 110 & 113 \\
\hline D & Ear lobe & 91 & 93 & 98 & 105 \\
\hline E & Ear lobe & 95 & 97 & 100 & 103 \\
\hline F & Finger web & 105 & 107 & 110 & 113 \\
\hline G & Finger web & 88 & 90 & 93 & 97 \\
\hline
\end{tabular}


Tables-1 and 2 given below shows the data collected from each person, during the experiment. Table-1 shows the fasting glucose value obtained from invasive laboratory test.Table-2 shows the variation in glucose concentration obtained from proposed non invasive device after meal.

For validation of the experiment, a value obtained from the proposed device is compared with invasive laboratory test result. Two non-diabetic persons were considered for this experiment. At first the device is calibrated using the fasting glucose value which is measured invasively. Then glucose concentration readings of the above two persons after meal, using the proposed device and invasive laboratory test, is taken at an interval of one hour.

\section{Data obtained from Person 1}

Invasively measured fasting glucose concentration of person 1: $83 \mathbf{~ m g} / \mathbf{d l}$.

Measurement site: finger web

Table-3: Data obtained from sensor and invasive laboratory test of person 1

\begin{tabular}{|l|l|l|l|}
\hline Sl.no & $\begin{array}{l}\text { Time } \\
(\mathrm{min})\end{array}$ & $\begin{array}{l}\text { Non invasive } \\
\text { glucose value from } \\
\text { sensor }(\mathrm{mg} / \mathrm{dl})\end{array}$ & $\begin{array}{l}\text { Invasive } \\
\text { laboratory test } \\
\text { result }\end{array}$ \\
\hline 1 & 60 & 114 & 98 \\
\hline 2 & 120 & 143 & 138 \\
\hline
\end{tabular}

\section{Data obtained from Person 2}

Invasively measured fasting glucose concentration of person 2: $85 \mathbf{~ m g} / \mathbf{d l}$.

Measurement site: finger web

Table -4: Data obtained from sensor and invasive laboratory test of person 2

\begin{tabular}{|l|l|l|l|}
\hline Sl.no & $\begin{array}{l}\text { Time } \\
(\mathrm{min})\end{array}$ & $\begin{array}{l}\text { Non invasive glucose } \\
\text { value from sensor } \\
(\mathrm{mg} / \mathrm{dl})\end{array}$ & $\begin{array}{l}\text { Invasive } \\
\text { laboratory test } \\
\text { result }\end{array}$ \\
\hline 1 & 60 & 91 & 89 \\
\hline 2 & 120 & 103 & 93 \\
\hline
\end{tabular}

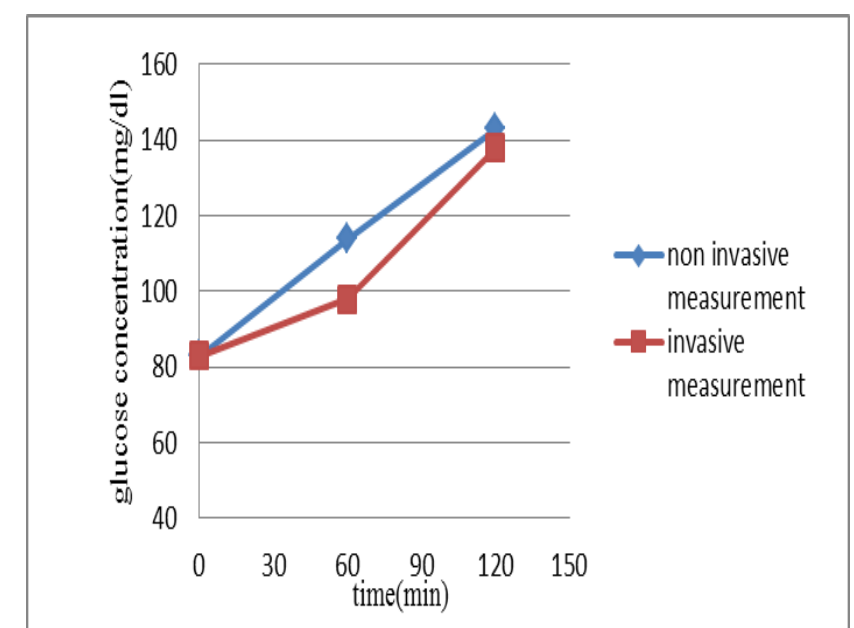

Fig-2: Noninvasive and invasive glucose measurement of person 1

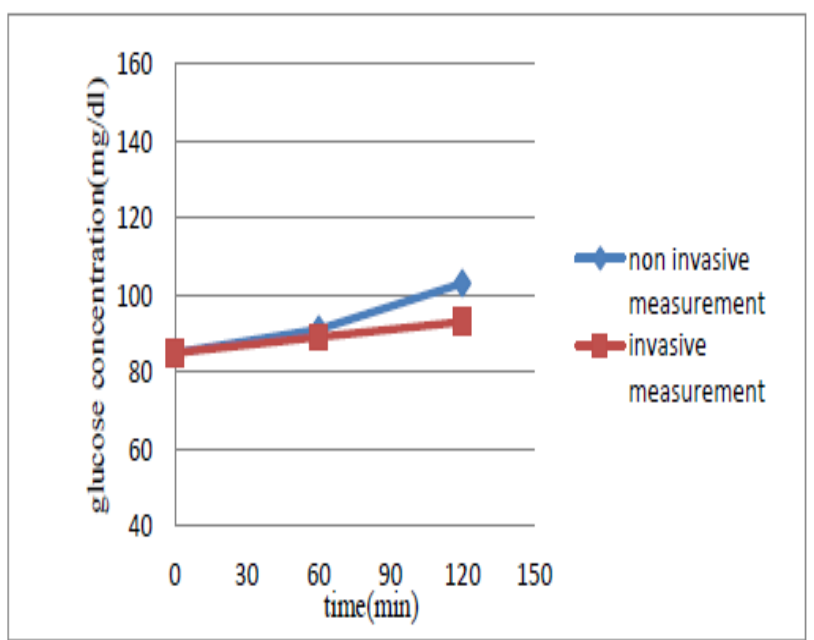

Fig-3: Non invasive and invasive glucose measurement of person 2

It was found from the figures 2 and 3, that the values acquired from this proposed device shows an incremental nature as compared with the blood glucose level obtained with invasive laboratory test.

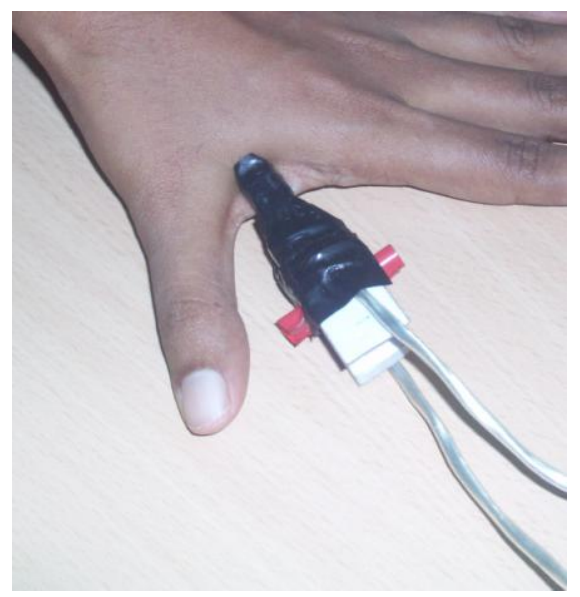

Fig-4: Emitter and detector setup

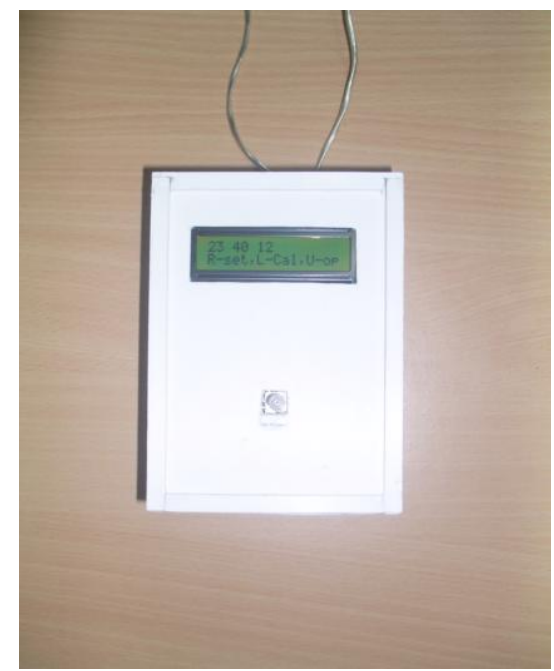

Fig-5: Final hardware 


\section{CONCLUSION}

In the present work an embedded system for noninvasive procedure based blood glucose measurement for conducting Glucose Tolerance Test is developed. By using this proposed device pricking can be reduced to one while conducting glucose tolerance test.Experiments are carried out using the proposed non invasive device and the results obtained are comparable with invasive measurement. This device is also useful for continuous glucose measurement of any bedridden or ICU patients.

Challenges faced in this work are:

i) The skin transmittance is different for different persons depending on the colour, texture of the skin and thickness of measurement site. So the proposed device needs to be calibrated for each person before conducting the test.

ii) The sensing unit must be properly positioned and not allowed to move during the entire measurement period.

In future the performance of the proposed system can be improved by adopting differential measurement in which the difference between incident light and transmitted light from skin is used for calculating glucose concentration.

\section{REFERENCES}

[1]. Omar S. Khalil, "Spectroscopic and Clinical Aspects of Noninvasive Glucose Measurements", Clinical Chemistry 45:2 165-177 (1999)

[2]. JyotiYadav, Asha Rani, Vijander Singh, Bhaskar Mohan Murari, "Near-infrared LED based Non-invasive Blood Glucose Sensor", 2014 international conference on Signal Processing and Integrated Networks (SPIN).

[3]. Ola S. Abdalsalam, Al-khanssa M. Osman, Roua M. Abd-Alhadi, Saad D. Alshmaa, " Design of Simple Noninvasive Glucose Measuring Device”,2013 International Conference on Computing, Electrical and Electronic Engineering (ICCEEE)

[4]. A.G.Pavithra, D.MeneshaKaran,D. Ajith Kumar, P .S. AnuShalin,"Non invasive technique to measure glucose and haemoglobin level in blood using NIR spectroscopy",International Journal of scientific research and management.Volume2,Issue4,2013 